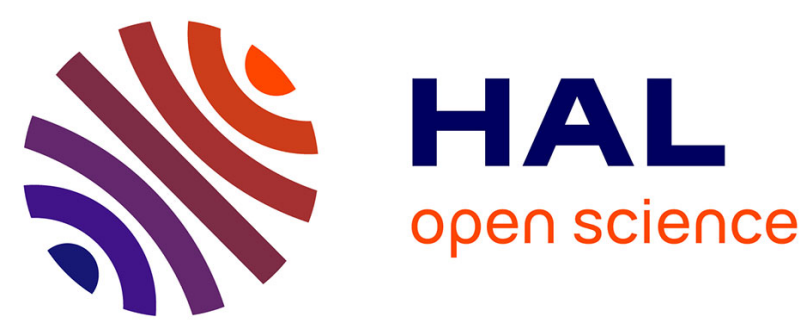

\title{
One-week in vivo sustained release of a peptide formulated into in situ forming implants
}

Marianne Parent, Igor Clarot, Sébastien Gibot, Marc Derive, Philippe Maincent, Pierre Leroy, Ariane Boudier

\section{To cite this version:}

Marianne Parent, Igor Clarot, Sébastien Gibot, Marc Derive, Philippe Maincent, et al.. One-week in vivo sustained release of a peptide formulated into in situ forming implants. International Journal of Pharmaceutics, 2017, 521 (1-2), pp.357-360. 10.1016/j.ijpharm.2017.02.046 . hal-01482708

\section{HAL Id: hal-01482708 https://hal.science/hal-01482708}

Submitted on 29 Mar 2017

HAL is a multi-disciplinary open access archive for the deposit and dissemination of scientific research documents, whether they are published or not. The documents may come from teaching and research institutions in France or abroad, or from public or private research centers.
L'archive ouverte pluridisciplinaire $\mathbf{H A L}$, est destinée au dépôt et à la diffusion de documents scientifiques de niveau recherche, publiés ou non, émanant des établissements d'enseignement et de recherche français ou étrangers, des laboratoires publics ou privés.

\section{(ㅇ)(1) $\$$}

Distributed under a Creative Commons Attribution - NonCommercial - NoDerivatives| 4.0 
1 One-week in vivo sustained release of a peptide formulated into in situ forming

4 Marianne PARENT ${ }^{a, *}$, Igor CLAROT ${ }^{\mathbf{a}}$, Sébastien GIBOT $^{\mathbf{b}}$, Marc DERIVE ${ }^{\mathrm{c}}$, Philippe

5 MAINCENT ${ }^{\text {a }}$, Pierre LEROY ${ }^{a}$, Ariane BOUDIER ${ }^{a}$

6

7 a Université de Lorraine, CITHEFOR, EA 3452, Nancy, France

$8 \quad{ }^{b}$ Université de Lorraine, INSERM U1116, Vandouvre-lès-Nancy, France.

$9 \quad{ }^{c}$ INOTREM, Vandouvre-lès-Nancy, France.

$10 *$ corresponding author: Dr Marianne Parent, Université de Lorraine, CITHEFOR, EA3452, 5 rue

11 A. Lebrun, BP 80403 F-54001 Nancy Cedex (France), phone (+33) 3727473 07,

12 marianne.parent@univ-lorraine.fr

13 GRAPHICAL ABSTRACT
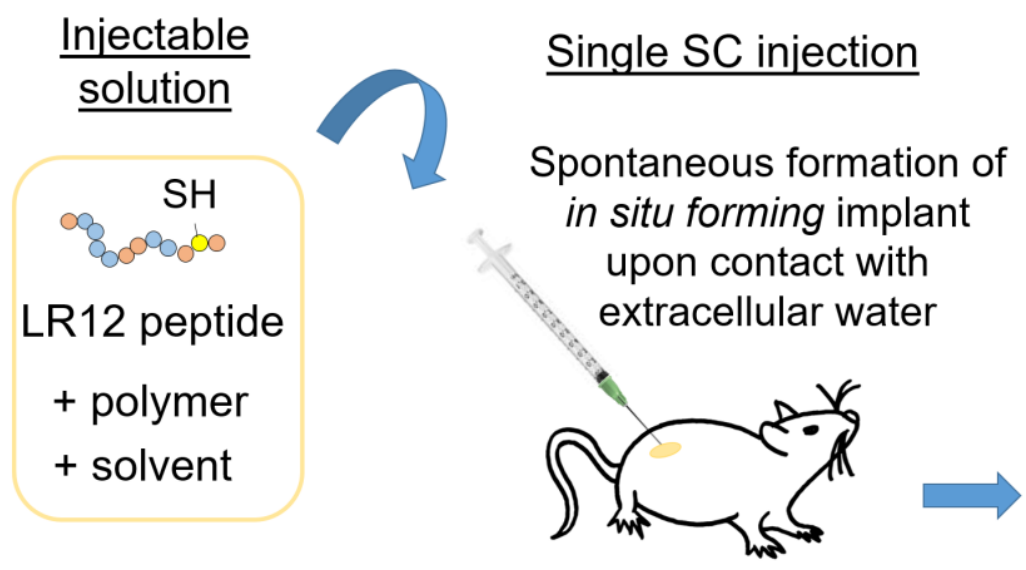

7-days sustained release

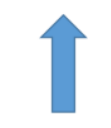

Stability

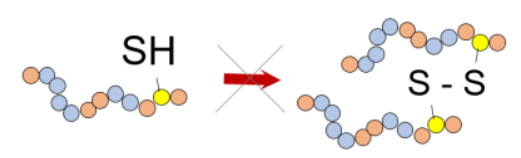




\section{ABSTRACT}

16 The LR12 peptide has been reported to reduce the size of infarct and improve both cardiac function

17 and survival in myocardial infarction in murine models, after daily repeated intraperitoneal

18 injections. In order to protect peptide from degrading and to prolong its release, in situ implants

19 based on biocompatible biodegradable polymers were prepared and both in vitro and in vivo

20 releases were evaluated after subcutaneous administration to Wistar rats. A progressive and

21 complete release was obtained in vitro in 3 weeks. In vivo, a 7-day sustained release was

22 demonstrated after administrating the formulation once; bioavailability was improved by

23 protecting the peptide against the degradation identified as a dimerization through disulfide bond

24 formation. As a conclusion, in situ forming formulations are a suitable alternative for the

25 therapeutic use of this peptide.

\section{KEYWORDS}

$28 \quad$ LR12

29 TREM-1 inhibitory peptide

30 Sustained delivery

31 In situ-forming depot

32 Bioavailability

33 Dimerization 


\section{TEXT}

36 With 17.3 million deaths per year (a number expected to grow to more than 23.6 million by 2030),

37 cardiovascular disease is the leading global cause of death. Related direct and indirect costs are

38 estimated to be higher than $\$ 320.1$ billion, including health expenditures and loss of productivity

39 (Mozaffarian et al. 2015). As the immune system and the inflammation are now recognized as key

40 players in the establishment and exacerbation of cardiovascular diseases, new therapeutic strategies

41 have been emerging to control them. In this context, the triggering receptor expressed on myeloid-

42 cells-1 (TREM-1) has been identified as an interesting target. This immune-receptor is expressed

43 by neutrophils, macrophages and mature monocytes, and acts as an amplifier of the innate immune

44 response during both infectious and sterile inflammation (Bouchon et al. 2001, Gibot et al. 2009,

45 Zhou et al. 2013). Recently, the modulation of TREM-1 signaling by an inhibitory dodecapeptide

46 (LR12, Figure 1A) reduced the size of infarct and improved both cardiac function and survival in

47 a murine model of myocardial infarction (Boufenzer et al. 2015). In that study, LR12 (5 mg/kg)

48 was intraperitoneally (IP) administered once a day for 5 days. This therapeutic scheme should be

49 advantageously replaced by a single injection of a sustained-release formulation. Moreover, LR12

50 oxidizes spontaneously in aqueous media with a short half-life ( $\left.\mathrm{t}_{1 / 2}\right)$ (approximately 14 hours in

51 phosphate buffer saline (PBS) in vitro, and 0.5 hour in blood ex vivo): a disulfide bridge is formed

52 between two peptides, generating a dimer (Figure 1B). While still to be confirmed by further

53 investigation, preliminary experiments on cells suggested that the dimer is devoid of

54 pharmacological activity. Consequently, the formulation should both sustain LR12 release for at

55 least a 5-day period and, as much as possible, protect the drug from degrading by dimerization.

56 In this contribution, the in vitro and in vivo release of LR12 (both the monomer and its main product 57 of degradation, i.e. the dimeric form) from in situ forming implants have been evaluated. In situ 
58 forming implants are liquid formulations which, when injected into aqueous environments,

59 precipitate as solid polymeric matrices entrapping the drug (Parent et al. 2013a). Sustained releases

60 obtained with in situ forming implants have been described in the literature for a wide range of

61 drugs with various physicochemical properties. Herein, poly-lactide-co-glycolide (PLGA,

62 Resomer RG502H, 50:50 ratio LA:GA) and poly-lactide (PLA, Resomer R203S) were used as

63 biocompatible biodegradable polymers due to their frequent use in in situ forming formulations

64 and their lack of toxicity. Polymers were solubilized $(18.7 \% \mathrm{w} / \mathrm{w})$ in triacetin $(\mathrm{TA}, 74.8 \% \mathrm{w} / \mathrm{w})$

65 before adding the drug $(6.5 \% \mathrm{w} / \mathrm{w})$. The concentration of peptide used in the formulation allowed

66 to subcutaneously inject a dose of $80 \mathrm{mg} \mathrm{LR} 12 / \mathrm{kg}$ to the animals. This can be compared to the total

67 dose of $25 \mathrm{mg} / \mathrm{kg}$ of free LR12 administered via several IP injections in the study, which

68 demonstrated the benefit of LR12 in myocardial infarction (Boufenzer et al. 2015). This dose is

69 compatible with a slow delivery of LR12 from the reservoir formulation during the time of

70 experiment, and it is likely without safety issues. Additionally, with this concentration, the

71 viscosity of the formulation remained suitable for an easy injection.

72 Formulations were prepared and in vitro release experiments were performed in physiological

73 buffer saline according to previously reported protocols (Parent et al. 2013b). An HPLC-UV

74 method was developed, then validated for selectivity, precision, accuracy and linearity according

75 to the FDA guidelines, and used to quantify LR12 (monomer and dimer) released in the aqueous

76 medium (Figure 2). The chromatographic system was the same as previously described (Parent et

77 al. 2016). The elution was isocratic $(86 / 14 \% \mathrm{v} / \mathrm{v}$ of water/acetonitrile $+0.1 \%$ trifluoroacetic acid)

78 with $20 \mu \mathrm{L}$ of injected sample. The detection was set at $220 \mathrm{~nm}$. Linearity was verified for

79 monomer and dimer between 1.0 and $50.0 \mu \mathrm{g} / \mathrm{mL}\left(\mathrm{y}=14.30 \mathrm{x}-17.55\right.$ and $\mathrm{R}^{2}=0.994$ for monomer,

$80 \mathrm{y}=14.15 \mathrm{x}-11.87$ and $\mathrm{R}^{2}=0.996$ for dimer). Peptides remaining in the implants were also 
81 quantified with the same method after being extracted (dissolution of implants in ethyl acetate then

82 liquid-liquid extraction with PBS added with $0.1 \%$ trifluoroacetic acid, recoveries of $102.5 \pm 6.2$

$83 \%$ for the monomer and $100.3 \pm 4.2 \%$ for the dimer, $n=3$ ).

85 In vitro experiments demonstrated a 3-week sustained release of LR12 monomer from PLGA

86 implants (Figure 2A), without any burst, as expected when using a solvent with low water

87 solubility such as TA (Camargo et al. 2013). LR12 dimer also appeared progressively in the

88 medium. The release profile from PLA implants was different (Figure 2B): the monomer release

89 reached a plateau after 3 days, while the concentration of the dimer increased until the end of the

90 experiment. Dimer appears, resulting from the degradation of monomer, which can occur either

91 inside the implant or in the aqueous medium after its release. In the conditions of the in vitro release

92 test, non-formulated LR12 monomer spontaneously oxidized within time to form the disulfide

93 dimer, with a short half-life of 14 hours. Moreover, at the end of the release experiments (after 3

94 weeks), remaining LR12 ( $<5.0 \%$ of the initial load) was extracted and was shown to be mainly

95 under the monomer form $(95.0 \pm 1.3 \%$ for PLGA and $93.4 \pm 1.1 \%$ for PLA). The very low

96 proportion of dimer inside the implants suggests that the degradation of LR12 likely occurs in the

97 release medium rather than inside the formulations. To conclude, in situ implants offered a

98 sustained in vitro release of LR12 monomer up to 3 weeks, while efficiently protecting the drug

99 from dimerization.

100

101 In a previous study, blood concentrations of LR12 (monomer and dimer) after intraperitoneal 102 injection $(5 \mathrm{mg} / \mathrm{kg})$ to male Wistar rats were monitored (Parent et al. 2016). The same protocol and 
103 method were applied to quantify LR12 in blood after one single subcutaneous injection of in situ

104 implants (80 mg LR12/kg) to male Wistar rats (280-350 g). Rats were subcutaneously injected with

105 in situ forming formulation prepared with PLGA or PLA (final dose of $80 \mathrm{mg}$ LR12/kg, $21 \mathrm{G}$

106 needle used for injection). Under isoflurane anesthesia, blood was collected 1h, 1, 3, 7 or 14 days

107 after the treatment, before sacrificing the animal and retrieving the implants for residual LR12

108 quantitation. Three animals were used for each time point for each polymer (total $=30$ rats). Blood

109 samples were treated and analyzed with the HPLC-fluorescence method as previously reported

110 (Parent et al. 2016). Remaining LR12 was also extracted from the retrieved implants and quantified

111 as described above for the in vitro study. At autopsy, no sign of irritation or inflammation of the

112 tissues surrounding the implants was observed, whatever the polymer used (Representative

113 photograph of extraction is supplied in Supplementary data S1).

114 After one single administration of in situ forming implants, LR12 monomer was detected at

115 therapeutic concentrations (between 100 and $400 \mathrm{ng} / \mathrm{mL}$ ) for a week (Figure 3). The area under

116 the concentration-time curve $\left(\mathrm{AUC}_{0 \rightarrow 7}\right.$ days $)$ was calculated using the trapezoidal rule and was

117 normalized by the received dose (Table 1). The highest observed plasma concentration $\left(\mathrm{C}_{\max }\right)$ and

118 the time required to reach $\mathrm{C}_{\max }\left(\mathrm{T}_{\max }\right)$ were obtained from the concentration-time curves. Results

119 indicated that in situ forming formulations significantly improved LR12 monomer bioavailability

120 by a factor 50, without any difference between PLGA and PLA. This can be explained by the

121 sustained-release properties of the in situ forming formulations, which increase the circulation time

122 of LR12, and by the protection of monomer from degradation because it was encapsulated into the

123 polymeric matrices. Although LR12 remaining inside the implants was mainly under the monomer

124 form, the proportion of dimer inside the in vivo implants increased within time ( $4.3 \pm 1.7 \%$ at day

1251 to $27.3 \pm 2.4 \%$ at day 7 for PLGA and $3.5 \pm 3.0 \%$ at day 1 to $26.1 \pm 10.9 \%$ at day 7 for PLA). 
126 After one week, more than $95 \%$ of the initial load was released. Compared with these in vivo

127 results, better LR12 protection (> 90\% remaining as monomer after 3 weeks in the implants) and

128 more sustained release (up to 3 weeks) with in situ forming implants were observed in vitro.

130 In the literature, only a few studies deal with in vivo peptide sustained delivery with in situ forming

131 formulations. A $48 \mathrm{~h}$ - in vivo release was for example reported for enfuvirtide, an anti-HIV fusion

132 inhibitor of 36 amino acids, when incorporated into implants made of PLGA and a mixture of

133 DMSO and triacetin (Kapoor et al. 2012). A sustained effect of $S$-nitrosoglutathione, a nitric oxide

134 donating tripeptide, was also observed over the same time length, using PLGA implants with $N$ -

135 methyl-2-pyrrolidone (NMP) as solvent (Parent et al. 2015). Regarding leuprolide acetate (9 amino

136 acids), a 14-days in vivo release in rats was described from PLGA/NMP implants (Mashayekhi et

137 al. 2013), but similar marketed formulations $\left(\right.$ Eligard $\left.^{\circledR}\right)$ allow therapeutic efficiency in humans

138 from 1 to up to 6 months after a single administration. In this study, in situ forming formulations

139 administered to healthy rats demonstrated their ability to deliver the dodecapeptide LR12 according

140 to a smooth and sustained profile at therapeutic concentrations for 7 days. As a result, this single

141 injection could be an interesting alternative to the current therapeutic scheme (free drug $5 \mathrm{mg} / \mathrm{kg}$,

142 daily injection for 5 days) proposed in myocardial infarction (Boufenzer et al. 2015). PLGA

143 formulations should probably be preferred over PLA ones, because they will be degraded faster,

144 while offering the same monomer bioavailability in this case, but with lower burst and less dimer

145 blood exposure (dimer AUC/dose increased by 12 for PLGA implants compared to IP

146 administration and by 18 for PLA ones, Table 1). Modifications of the peptide itself or of the

147 formulation could also be envisaged to further increase the in vivo duration of release. For example,

148 the PEGylation of a natural polysaccharide enhanced the in vivo mean retention time from $1.0 \mathrm{~h}$ to 
1492.8 days, and this result was drastically improved (up to 13 days) when the conjugate was

150 formulated into PLGA in situ implants (Shi et al. 2014, 2015).

151 To conclude, this study demonstrates that in situ formulations are promising candidates for the

152 therapeutic use of LR12, a TREM-1 inhibitory dodecapeptide useful in many conditions involving

153 inflammation and exacerbated immune response. However, pharmacokinetics of LR12 could be

154 modified in pathological situations, for example by the apparition of the soluble form of TREM-1

155 in the blood (Boufenzer et al. 2015). The benefit of in situ formulations for LR12 treatment should

156 therefore be confirmed in animals suffering from myocardial infarction for example.

\section{ACKNOWLEDGEMENTS}

159 This work was supported by the "Fondation pour la Recherche Médicale", grant number 160 DBS20131128445, to Philippe Maincent.

161 Authors thank F. Dupuis and M.L. Bouressam for their assistance during blood sampling. They

162 also acknowledge M. Alcazar-Duque, M. Girardon and C. Verebi for their contribution in in vitro 163 release experiments and HPLC-UV validation. They are also very grateful to Marjorie Antoni 164 (lifelong learning division of Université de Lorraine) for helping to improve the level of English in 165 the manuscript.

167 REFERENCES

168 Bouchon, A., Facchetti, F., Weigand, M.A., Colonna, M., 2001. TREM-1 amplifies inflammation 169 and is a crucial mediator of septic shock. Nature 410, 1103-1107. 
171 Boufenzer, A., Lemarié, J., Simon, T., Derive, M., Bouazza, Y., Tran, N., Maskali, F., Groubatch,

172 F., Bonnin, P., Bastien, C., Bruneval, P., Marie, P.Y., Cohen, R., Danchin, N., Silvestre, J.S., Ait-

173 Oufella, H., Gibot, S., 2015. TREM-1 mediates inflammatory injury and cardiac remodeling

174 following myocardial infarction. Circ. Res. 116, 1772-1782.

175

176 Camargo, J., Sapin, A., Nouvel, C., Daloz, D., Leonard, M., Bonneaux, F., Six, J.L., Maincent, P., 177 2013. Injectable PLA-based in situ forming implants for controlled release of ivermectin, a BCS 178 class II drug: solvent selection based on physico-chemical characterization. Drug Dev. Ind. Pharm. $17939,146-155$.

180

181 Gibot, S., Massin, F., Alauzet, C., Derive, M., Montemont, C., Collin, S., Fremont, S., Levy, B., 182 2009. Effects of the TREM 1 pathway modulation during hemorrhagic shock in rats. Shock 32, $183 \quad 633-637$.

185 Kapoor, D.N., Katare, O.M., Dhawan, S, 2012. In situ forming implant for controlled delivery of 186 an anti-HIV fusion inhibitor. Int. J. Pharmaceut. 426, 132-143.

188 Mashayekhi, R., Mobedi, H., Najafi, J., Enayati, M., 2013. In vitro/in vivo comparison of 189 leuprolide acetate release from an in situ forming PLGA system. DARU J. Pharm. Sci. 21:57, 190 doi:10.1186/2008-2231-21-57

192 Mozaffarian, D., Benjamin, E.J., Go, A.S., Arnett, D.K., Blaha, M.J., Cushman, M., de Ferranti, 193 S., Despres, J-P., Fullerton, H.J., Howard, V.J., Huffman, M.D., Judd, S.E., Kissela, B.M., 
194 Lackland, D.T., Lichtman, J.H., Lisabeth, L.D., Liu, S., Mackey, R.H., Matchar, D.B., McGuire,

195 D.K., Mohler, E.R. 3rd, Moy, C.S., Muntner, P., Mussolino, M.E., Nasir, K., Neumar, R.W.,

196 Nichol, G., Palaniappan, L., Pandey, D.K., Reeves, M.J., Rodriguez, C.J., Sorlie, P.D., Stein, J.,

197 Towfighi, A., Turan, T.N., Virani, S.S., Willey, J.Z., Woo, D., Yeh, R.W., Turner, M.B., on behalf

198 of the American Heart Association, Statistics Committee and Stroke Statistics Subcommittee,

199 2015. Heart disease and stroke statistics-2015 update: a report from the American Heart

200 Association. Circulation 131, e29-e322.

201

202 Parent, M., Nouvel, C., Koerber, M., Sapin, A., Maincent, P., Boudier, A., 2013a. PLGA in situ 203 implants formed by phase inversion: critical physicochemical parameters to modulate drug release.

204 J. Control. Release 172, 292-304.

206 Parent, M., Boudier, A., Dupuis, F., Nouvel, C., Sapin, A., Lartaud, I., Six, J.L., Leroy, P., 207 Maincent, P., 2013b. Are in situ formulations the keys for the therapeutic future of S-nitrosothiols? 208 Eur. J. Pharm. Biopharm. 85, 640-649.

210 Parent, M., Boudier, A., Maincent, P., Gibot, S., Ait-Oufella, H., Boufenzer, A., Jolly, L., Derive,

211 M., Kouach, M., Goossens, J.F., Leroy, P., Clarot, I., 2016. LR12-peptide quantitation in whole 212 blood by RP-HPLC and intrinsic fluorescence detection: validation and pharmacokinetic study.

213 Biomed. Chromatogr., doi: 10.1002/bmc.3877.

215 Parent, M., Boudier, A., Perrin, J., Vigneron, C., Maincent, P., Violle, N., Bisson, J.F., Lartaud, I., 216 Dupuis, F., 2015. In situ microparticles loaded with S-nitrosoglutathione protect from stroke. PLoS 217 One 10, e0144659. 
219 Shi, X., Lin, X., Zheng, X.W., Feng, Y., Shen, L., 2014. Injectable long-acting systems for Radix

220 Ophiopogonis polysaccharide based on mono-PEGylation and in situ formation of a PLGA depot.

221 Int. J. Nanomed. 9, 5555-5563.

223 Shi, X., Lin, X., Yao, C.X., Shen, L., Feng, Y., 2015. Injectable long-acting in situ forming systems

224 for Radix Ophiopogonis polysaccharide. Int. J. Biol. Macromol. 72, 553-559.

226 Zhou, J., Chai, F., Lu, G., Hang, G., Chen, C., Chen, X., Shi, J., 2013. TREM-1 inhibition attenuates

227 inflammation and tumor within the colon. Int. Immunopharmacol. 17, 155-161.

\section{FIGURES}

A. $\quad$ Hydrophobic amino acid $\quad$ Acidic amino acid (charge $<0$ at neutral $\mathrm{pH}$ )

OHydrophilic amino acid $\bigcirc$ Thiol-containing amino acid

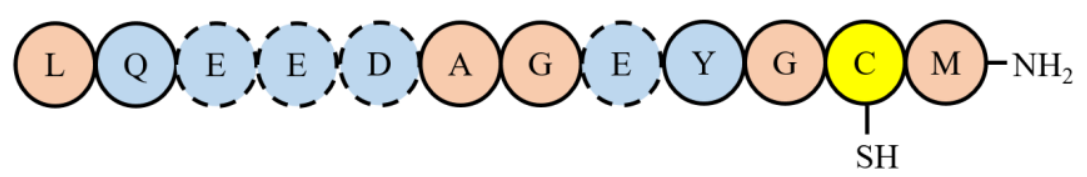

B.
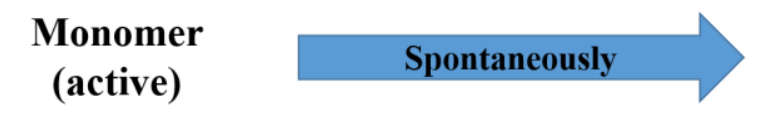

Dimer

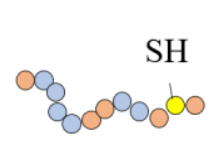

$\mathrm{T}_{1 / 2}=14$ hours in PBS
$\mathrm{T}_{1 / 2}=0.5$ hour in rat blood (inactive)

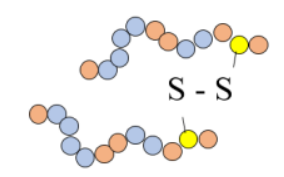

231 Figure 1: A) Murine sequence of LR12 monomer peptide (Mr $1341 \mathrm{~g} / \mathrm{mol}$, calculated pI 3.6) 232 and B) main pathway of degradation by dimerization. 


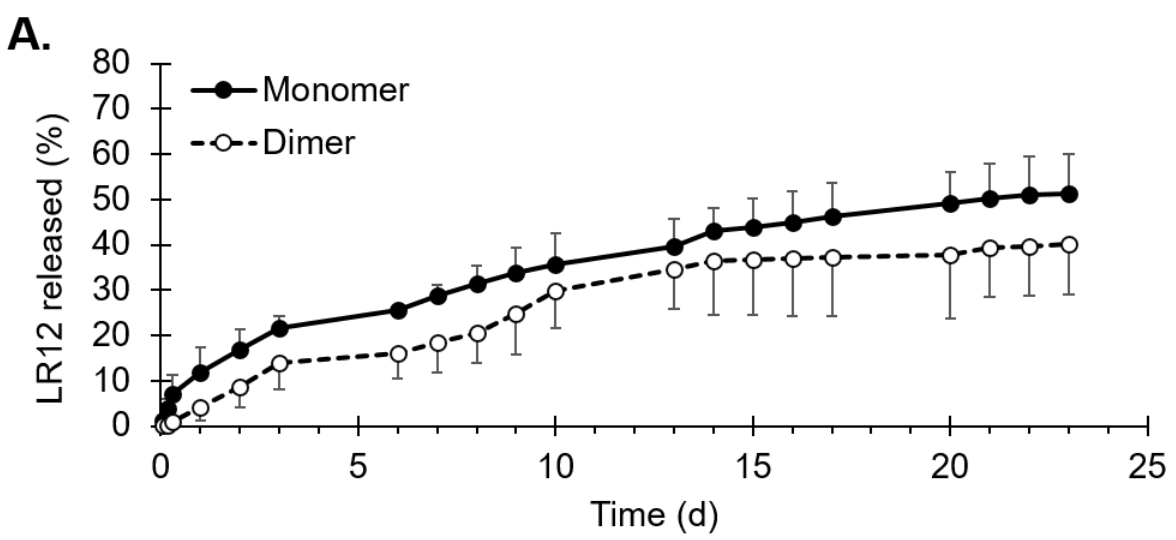

B.

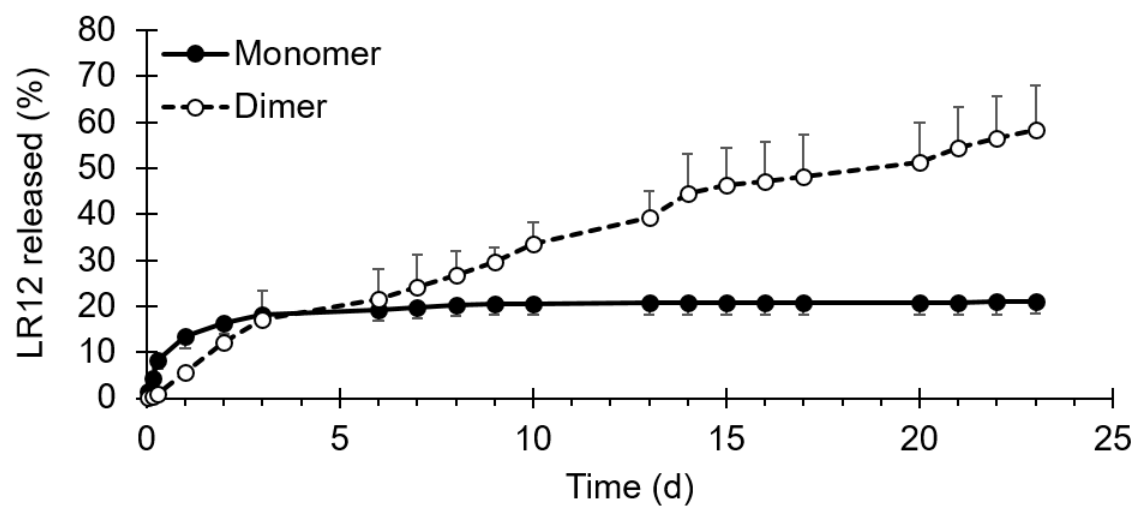

234 Figure 2: In vitro release profiles of LR12 (monomer and dimer) obtained from in situ implants 235 made of TA and of either PLGA (A) or PLA (B). Results are presented as mean \pm sd of three 236 experiments. 

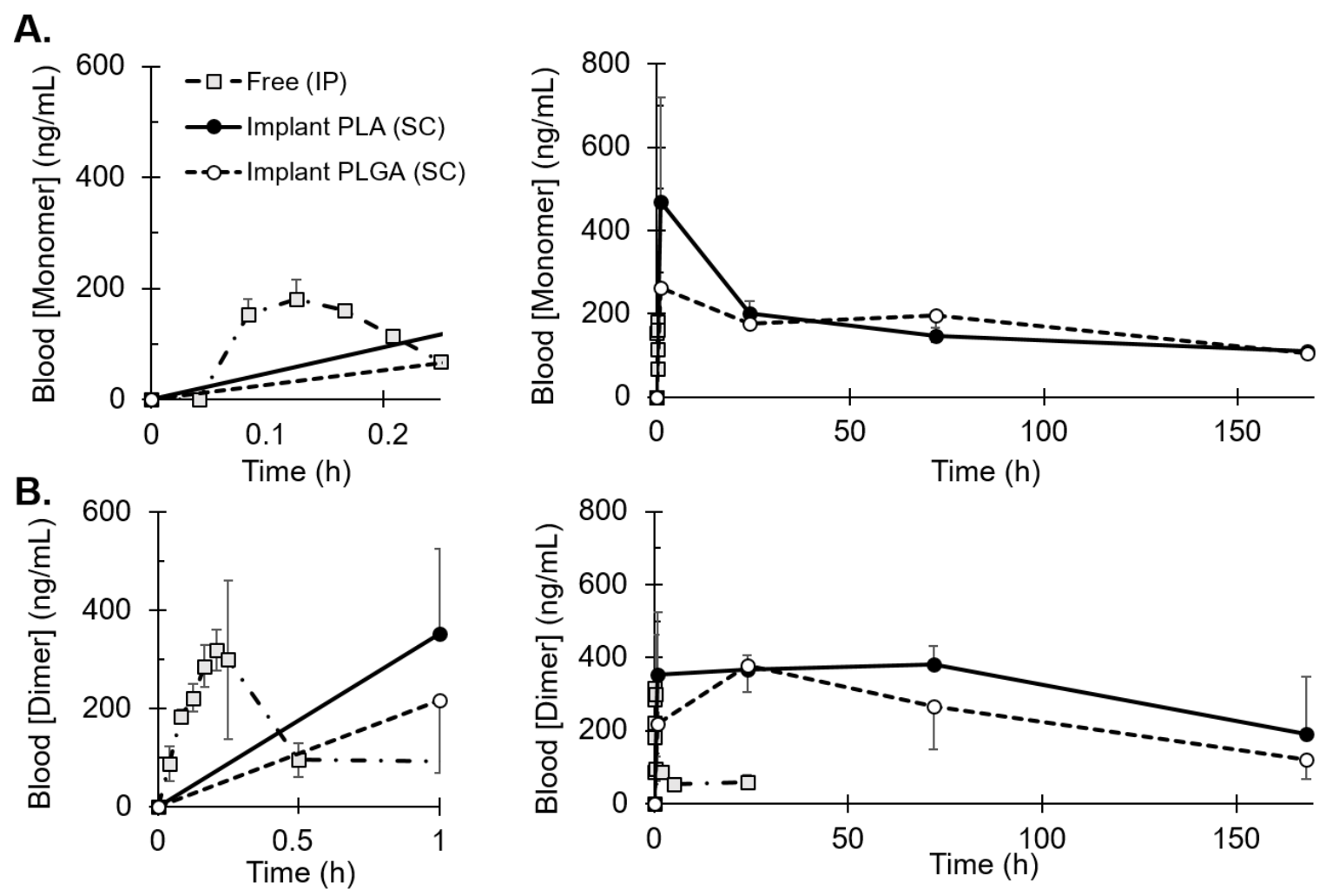

239 Figure 3: Blood concentrations of LR12 (monomer form, panel A and dimer form, panel B) after 240 administration of LR12 monomer (unformulated, $5 \mathrm{mg} / \mathrm{kg}$, intraperitoneally $v s$ formulated into in 241 situ implants, $80 \mathrm{mg} / \mathrm{kg}$, subcutaneously). Limit of quantification of the method was $50 \mathrm{ng} / \mathrm{mL}$. All 242 experiments were performed in accordance with the European Community guidelines 243 (2010/63/EU) for the use of experimental animals, and protocols were approved by the regional 244 and national ethical committees (project "Slow-release", APAFIS\#1146-2015071313458604 v3). 


\section{TABLE 1}

246 Pharmacokinetic parameters of LR12 after administration to male Wistar rats.

247

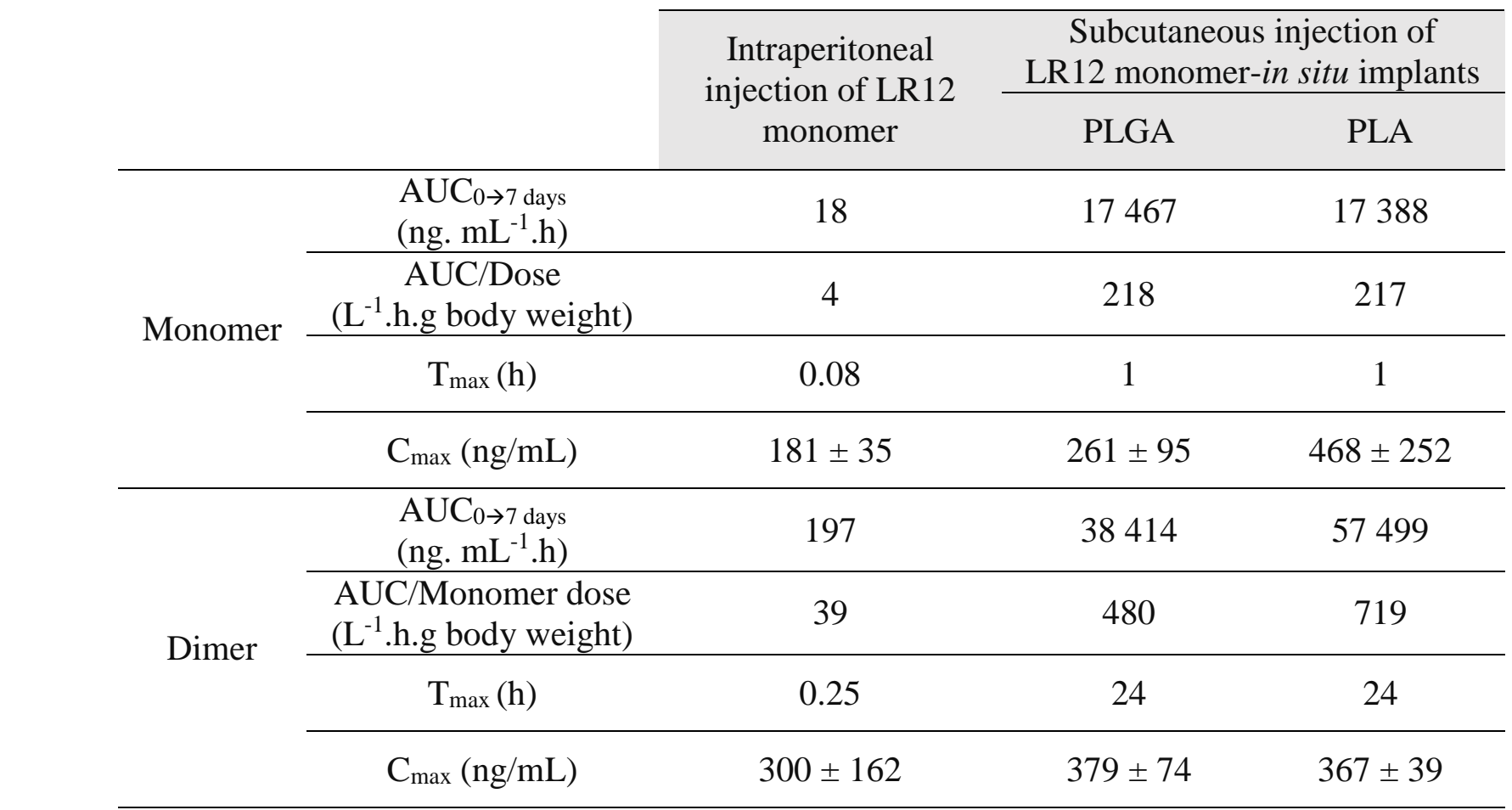

249 Supplementary data

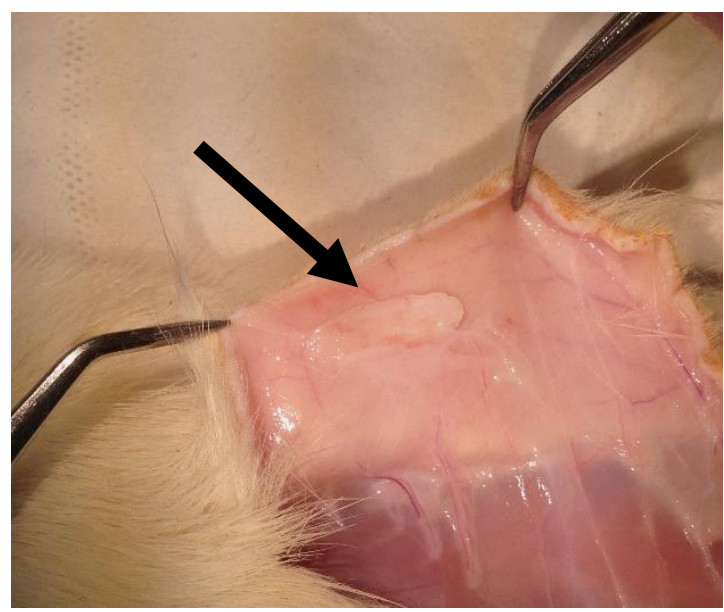

251 (S1): Representative photograph of the extraction of one in vivo in situ forming implant (here, PLA

252 implant, one week after injection). 